\title{
Separation of plasmid DNA isoforms using centrifugal ultrafiltration
}

\author{
Ehsan Espah Borujeni and Andrew L. Zydney \\ Department of Chemical Engineering, Pennsylvania State University, University Park, PA, USA
}

BioTechniques 53:49-56 (July 2012) doi 10.2144/0000113890

Keywords: DNA; centrifugal ultrafiltration; plasmid isoforms; supercoiled DNA

Supplementary material for this article is available at www.BioTechniques.com/article/113890

Centrifugal ultrafiltration is a well-established method for concentrating and purifying DNA. Here, we describe the use of centrifugal ultrafiltration for the separation of plasmid DNA isoforms based on differences in elongational flexibility of the supercoiled, open-circular, and linear plasmids. Transmission of each isoform is minimal below a critical value of the filtration velocity, which is directly related to the magnitude of the centrifugal speed and the system geometry. A discontinuous diafiltration process was used to enrich the desired isoform, as determined by agarose gel electrophoresis. The simplicity and efficacy of this membrane-based separation are attractive for multiple applications requiring the use of separated DNA isoforms.

Centrifugal ultrafiltration (UF) is used extensively for laboratory-scale purification, concentration, and isolation of a variety of biomolecules. This includes the use of centrifugal UF for rapid concentration of Legionella antigen in urine (1), the isolation of free benzalkonium chloride (BAC) molecules from micelle solutions (2), the analysis of low-molecular weight proteins in the human plasma proteome (3) and saliva (4), and the analysis of plant extracts (5).

Centrifugal UF is also a well-established method for concentrating and purifying DNA. Krowczynska and Henderson (6) described the use of centrifugal UF for the removal of unincorporated nucleotides and PCR primers after DNA amplification by the polymerase chain reaction. Schratter et al. (7) summarized the use of centrifugal UF in the construction of cosmid libraries (hybrid plasmids containing cos sequences), removal of restriction enzymes, and concentration of RNA. Centrifugal UF is also widely used in forensics laboratories for isolation and concentration of genomic DNA (8).

All of these applications of centrifugal UF involve recovery of large DNA in the retentate, with smaller molecules, impurities, and buffer components passed through the membrane and into the filtrate. However, recent work (9-11) has demonstrated that large DNA molecules with lengths of 3-17 kb (kilobase pairs) can actually be transmitted through the small pores in traditional ultrafiltration membranes under certain conditions because of flow-induced elongation of the DNA. This opens up exciting opportunities for DNA purification, e.g., for removal of large cell debris, viruses, or bacteriophage, with the DNA recovered in the filtrate. In addition, Latulippe and Zydney (12) demonstrated that pressuredriven ultrafiltration could also be used to separate the linear, supercoiled, and open circular isoforms of a given plasmid based on differences in elongational flexibility of these topological isoforms.

The objective of this work was to demonstrate the feasibility of using centrifugal UF for DNA separation, including removal of unwanted plasmid isoforms. Initial experiments were performed using the individual isoforms to identify the critical centrifugal conditions for retention and transmission of the plasmid. DNA separations were then performed using commercially available centrifugal UF devices, with the composition of the DNA in the filtrate and retentate analyzed by agarose gel electrophoresis (AGE).

\section{Materials and methods}

Experiments were performed using linear, open circular, and supercoiled isoforms of a $9.8 \mathrm{~kb}$ plasmid in which a $6.84 \mathrm{~kb}$ fragment was inserted into the SalI site of the pBluescript II KS+ plasmid vector. Stock solutions of the supercoiled plasmid were provided by Dr. Jeff Chamberlain at the University of Washington. The linear and open circular isoforms were obtained by enzymatic digestion using $2 \mathrm{U} / \mu \mathrm{g}$ of respectively $K p n \mathrm{I}$ and $\mathrm{Nt}$.A $\mathrm{AlwI}$ enzymes (New England Biolabs, Ipswich, MA, USA). BSA and the recommended buffer were added to $27.5 \mu \mathrm{g}$ of supercoiled plasmid DNA with the final volume of the digestion solution adjusted to $275 \mu \mathrm{L}$ by addition of deionized water obtained from a Barnstead Nanopure water purification system (Thermo Scientific, Dubuque, IA, USA) with a resistivity greater than $18 \mathrm{M} \Omega$. The digestion mixtures were incubated at $37^{\circ} \mathrm{C}$ for 3 $\mathrm{h}$ and then purified using a QIAquick PCR purification kit (QIAGEN, Valencia, CA, USA) which uses a silicagel membrane to bind the DNA in high-salt buffer while the impurities are washed away. The DNA was then eluted with low-salt Tris-EDTA (TE) buffer made by diluting a TE concentrate 


\section{BioTechniQues}

\section{Wionithly}

Issues Now

Availlable

for ipad and

iphone in

the firunes

App Store

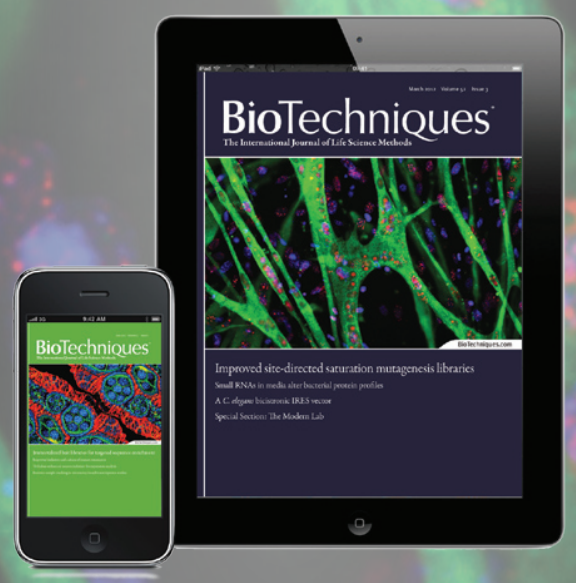

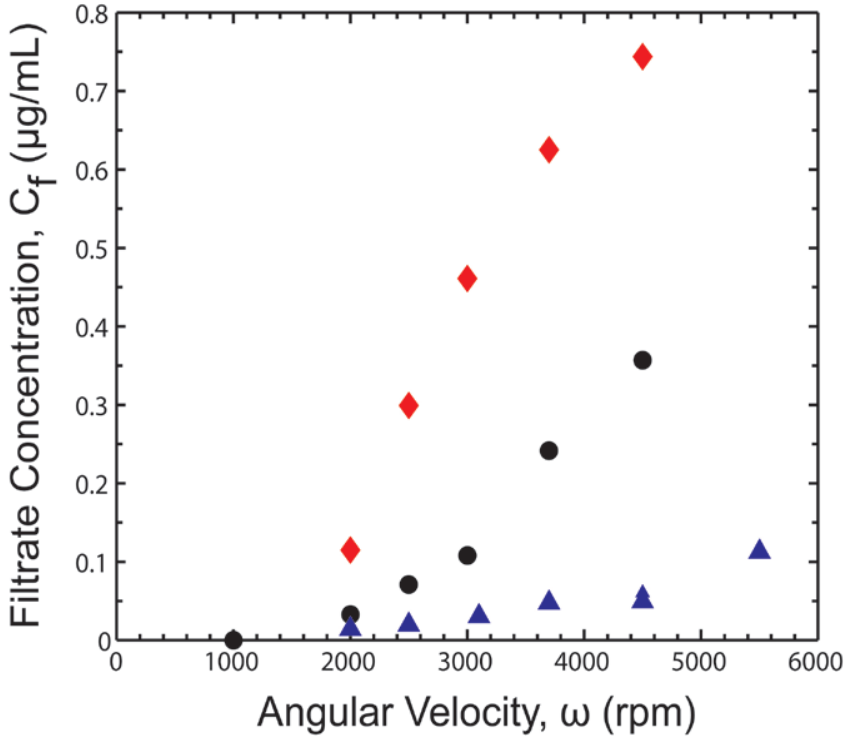

Figure 1. Centrifugal ultrafiltration of plasmids. Filtrate concentrations of the linear $(\diamond)$, supercoiled $(\bullet)$, and open circular $(\boldsymbol{\Delta})$ isoforms of a $9.8 \mathrm{kbp}$ plasmid.

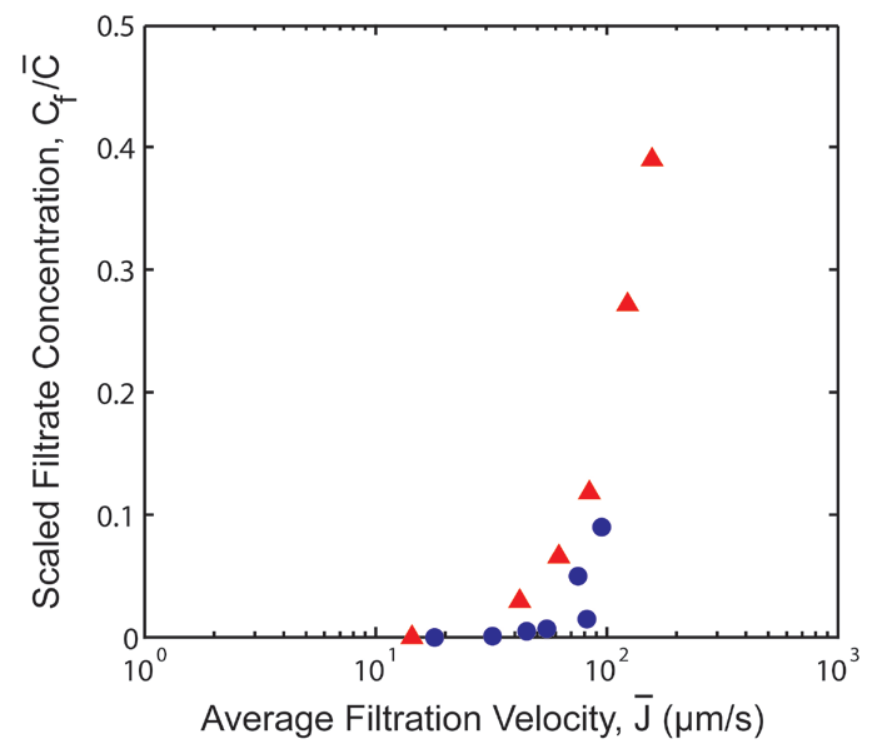

Figure 2. Centrifugal vs. pressure-driven ultrafiltration. Comparison of the scaled filtrate concentrations in centrifugal $(\boldsymbol{\Delta})$ and pressure-driven $(\bullet)$ UF.
(Sigma Aldrich, St. Louis, MO, USA) with deionized water. The final concentrations were measured by a NanoDrop 2000c UV spectrophotometer (Thermo Scientific) using the absorbance at 260 $\mathrm{nm}$. The purified plasmid solutions were stored at $-20^{\circ} \mathrm{C}$ and warmed to room temperature immediately prior to use.

Experiments were performed using NanoSep centrifugal UF tubes obtained from Pall Corporation (Ann Arbor, MI, USA). Each tube contains a feed reservoir with an encapsulated Omega polyethersulfone ultrafiltration membrane with an effective filtration area of $0.28 \mathrm{~cm}^{2}$ and a nominal molecular weight cutoff of $100 \mathrm{kDa}$. Membranes were initially flushed with $450 \mu \mathrm{L}$ of $0.05 \mathrm{~N} \mathrm{NaOH}$ followed by $1 \mathrm{~mL}$ of deionized water. A 5424 microcentrifuge (Eppendorf, USA) with a fixed-angle rotor (FA 45-24-11) of $45^{\circ}$ was used for centrifugation. The centrifugal speed could be varied up to $15,000 \mathrm{rpm}$, which corresponds to approximately $16,520 \times \mathrm{g}$ (for a centrifuge tube filled with $450 \mu \mathrm{L}$ of liquid) based on the rotor arm length of $0.066 \mathrm{~m}$. 
The NanoSep centrifuge tube was initially filled with 450 $\mu \mathrm{L}$ of the feed, the rotation speed was set, and the tube was spun for a given period of time. The tube was then removed from the microcentrifuge, with the filtrate collected and weighed using a MS104S analytical balance (Mettler Toledo, Columbus, $\mathrm{OH}$, USA) with an accuracy of $10^{-4} \mathrm{~g}$. A small sample of the filtrate and retentate were collected for analysis of the plasmid concentration as described subsequently.

Multistep discontinuous diafiltration experiments were performed as follows. The NanoSep centrifuge tube was prepared as above and a centrifugal UF was performed to reduce the volume from $450 \mu \mathrm{L}$ to approximately $50 \mu \mathrm{L}$. The tube was then removed from the centrifuge with the filtrate collected and weighed. The retentate remaining within the tube was then diluted with additional TE buffer back to the initial volume. This entire procedure was repeated multiple times using the same centrifugal conditions, with the filtrate removed at the end of each UF step. A final sample was obtained from the retentate sample at the end of the experiment.

The total DNA concentration was evaluated using Quant-iT PicoGreen dsDNA assay kit (Life technologies). $75 \mu \mathrm{L}$ samples of the DNA solution were loaded into the wells of a 96-well GENiosFL microplate reader (TECAN, Durham, NC, USA) along with an equal volume of the PicoGreen reagent that was first diluted 200:1 with analytical TE buffer (Life Technologies). Samples were mixed for $180 \mathrm{~s}$ at $36^{\circ} \mathrm{C}$ using an orbital shaker, with the fluorescence intensity measured at $535 \mathrm{~nm}$. Actual concentrations were determined by comparison with a calibration curve constructed using known standards.

The composition of solutions containing mixtures of the different isoforms was determined by AGE. A $0.7 \%$ (w/w) agarose solution was prepared by dissolving $0.98 \mathrm{~g}$ of agarose powder (EMD Chemicals Inc., Gibbstown, NJ, USA) in $140 \mathrm{~mL}$ of diluted 10X Tris-Acetate-EDTA (TAE) buffer (Mediatech Inc., Manassas, VA, USA). An agarose gel was cast in an Owl B2 EasyCast mini gel electrophoresis system (Thermo Scientific) using a 12-tooth comb (B2-12-EA) with tooth thickness of $1.5 \mathrm{~mm}$. Each well was loaded with $18 \mu \mathrm{L}$ of the DNA mixture with $6 \times$ loading dye. A 1 -kb DNA ladder (New England BioLabs) was loaded into at least one well as a reference. An electric field of 70-75 V was applied with a PowerPac Basic power supply (Bio-Rad, Hercules, CA, USA) for $2-2.5 \mathrm{~h}$. The voltage was removed after sufficient time, and the gel was stained in diluted $10,000 \times$ SYBR Gold nucleic acid gel stain solution (Life Technologies) for 60-70 min. Gels were imaged using a Fluorchem FC2 image system (ProteinSimple, Santa Clara, CA, USA).

\section{Results and discussion}

Figure 1 shows typical experimental data obtained using NanoSep centrifugal UF tubes fitted with an Omega polyethersulfone ultrafiltration membrane with nominal molecular weight cut-off of $100 \mathrm{kDa}$. The tubes were initially filled with $450 \mu \mathrm{L}$ of the supercoiled, linear, or open circular isoform of a $9.8 \mathrm{~kb}$ plasmid suspended in $10 \mathrm{mM} \mathrm{Na}$ EDTA Tris buffer containing $10 \mathrm{mM} \mathrm{NaCl}$ at a concentration of $1 \mu \mathrm{g} / \mathrm{mL}$. Each data point represents results from a separate centrifugation. In each case, the centrifugation time was adjusted to collect $160 \pm 10 \mu \mathrm{L}$ of filtrate based on the model presented in the Supplementary materials. The y-axis shows the concentration of the plasmid isoform collected in the filtrate solution for each centrifugation, determined using the measured fluorescence of the PicoGreen stain. The $x$-axis is the set value of the angular

\section{Prove \& Publish Cell Line Authentication}

\author{
- STR Analysis of Human DNA
}

- Contamination Detection

- Species Determination

- Mycoplasma

- High Volume Laboratory

- Results in 5 Days

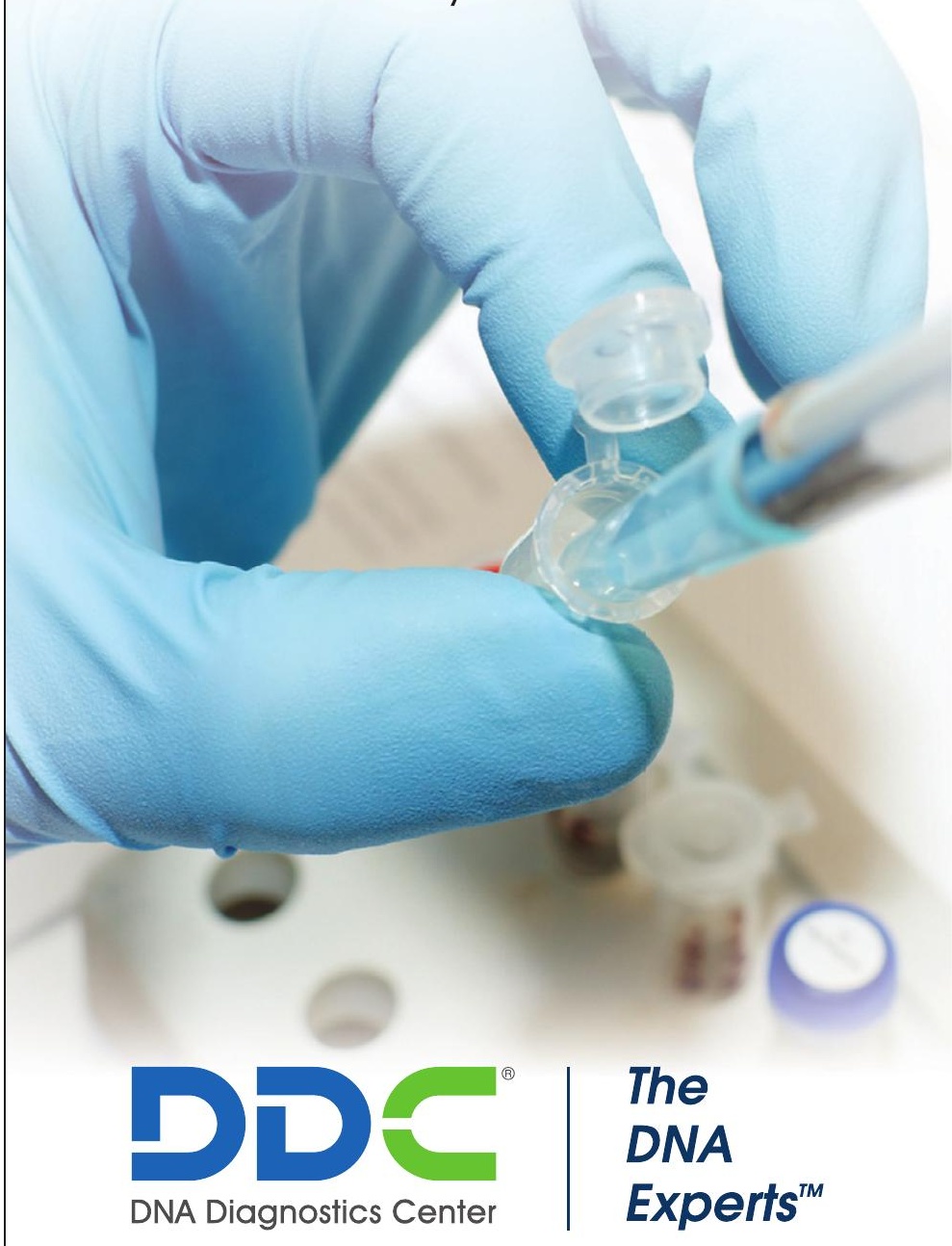

www.DNAcenter.com/cell-line
1-800-831-9830 
A)

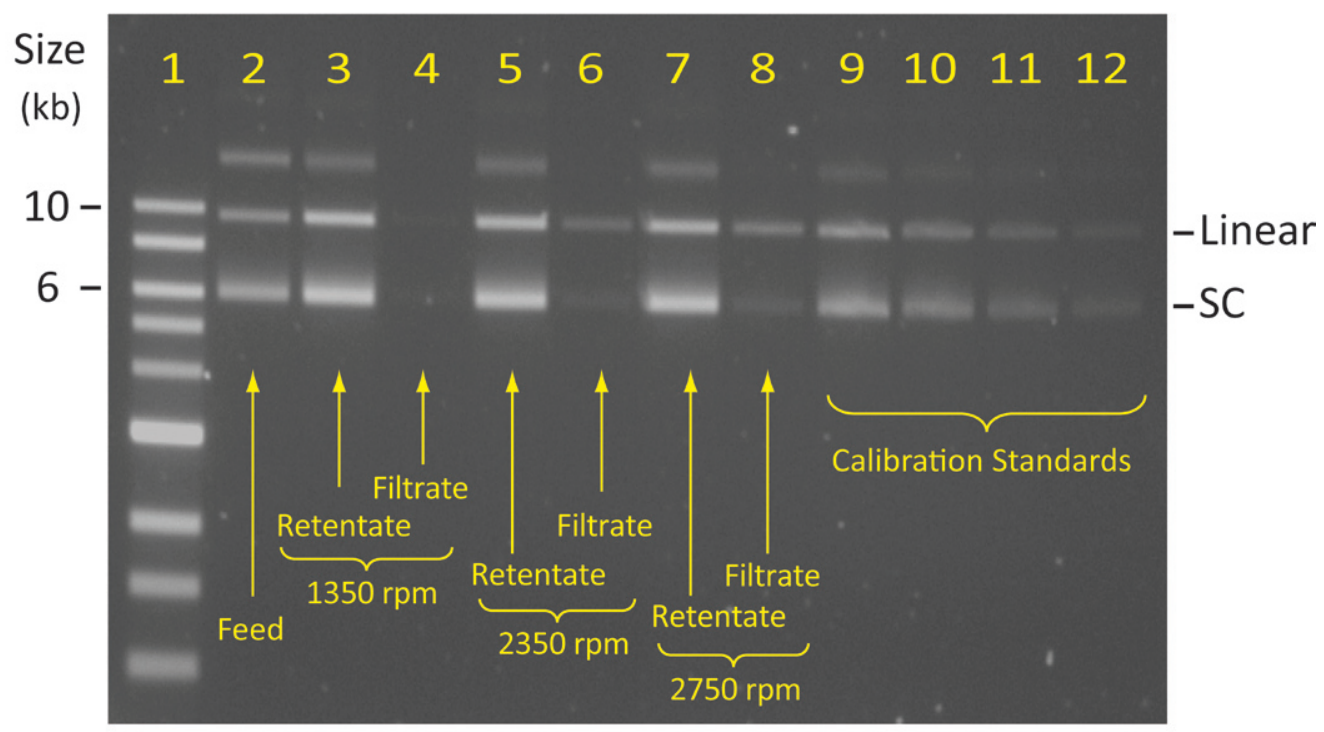

B)

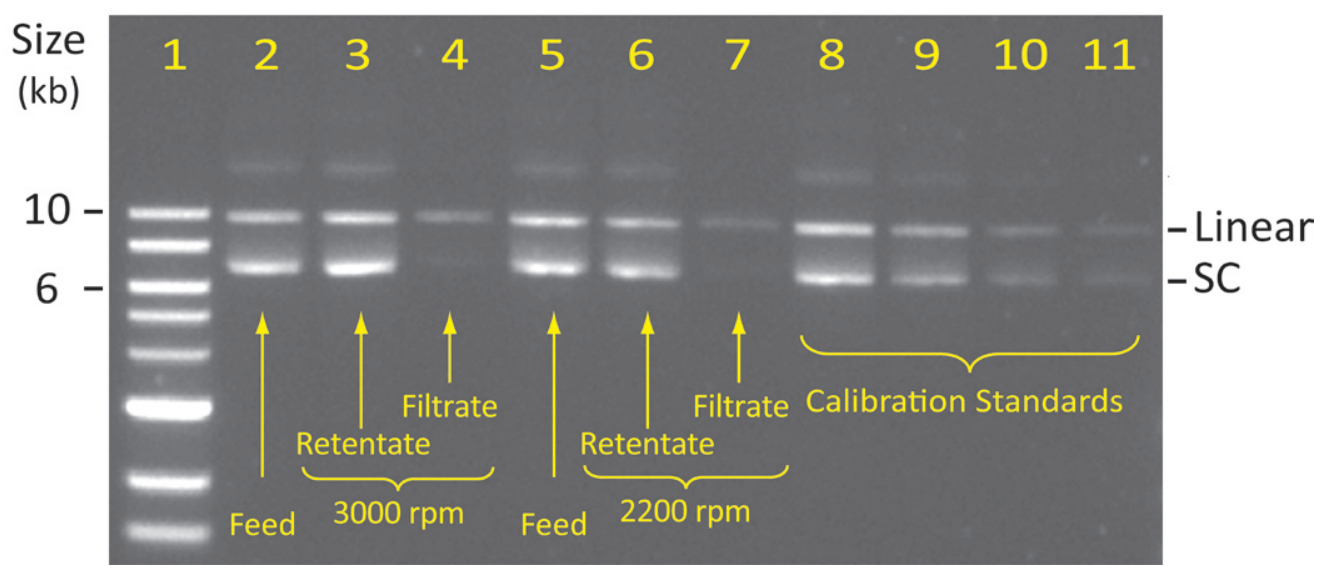

Figure 3. Separation of supercoiled and linear isoforms. Agarose gel electrophoregrams for centrifugal UF of the supercoiled (SC) and linear isoforms showing filtrate after collection of (A) $160 \pm 10 \mu \mathrm{L}$ and (B) $370 \pm 10 \mu \mathrm{L}$.

velocity used for the centrifugation. At any given angular velocity, the filtrate concentration for the linear isoform was significantly greater than that of the supercoiled isoform, with relatively little of the open circular isoform transmitted through the membrane out to even 5500 rpm.

The data for the supercoiled isoform show no measurable DNA in the filtrate (concentration $\leq 0.03 \mu \mathrm{g} / \mathrm{mL}$ ) for angular velocities below about $1500 \mathrm{rpm}$, consistent with complete retention of the $9.8 \mathrm{~kb}$ plasmid by the small pores of the $100 \mathrm{kDa}$ membrane under these conditions. Plasmid transmission increased with increasing centrifugal speed, with greater than $0.35 \mu \mathrm{g} / \mathrm{mL}$ (corresponding to $64 \mathrm{ng}$ ) of the supercoiled plasmid collected in the filtrate for centrifu- gation at $4500 \mathrm{rpm}$. This high degree of plasmid transmission occurs even though the radius of gyration of the supercoiled $9.8 \mathrm{~kb}$ plasmid is $117 \pm 3 \mathrm{~nm}$ in a 10 $\mathrm{mM} \mathrm{Na}$ EDTA buffer containing 200 $\mathrm{mM} \mathrm{Na}{ }^{2} \mathrm{Cl}$ (9), which is approximately $20 \times$ larger than the $6.4 \mathrm{~nm}$ pore size of a typical $100 \mathrm{kDa} U \mathrm{UF}$ membrane (9). The transmission of the linear DNA isoform was even greater, with a filtrate concentration of $0.75 \mu \mathrm{g} / \mathrm{mL}$ at $4500 \mathrm{rpm}$, even though the linear isoform has a radius of gyration of more than $200 \mathrm{~nm}$ (9).

There was no evidence of any plasmid degradation during the centrifugal UF; agarose gel electrophoresis of samples obtained from the initial feed, final retentate, and filtrate solutions showed bands corresponding to the linear, supercoiled, and open-circular plasmids as expected. As previously discussed, the high degree of plasmid transmission through the small membrane pores is because of the flow-induced elongation of the plasmid in the converging flowfield into the membrane pores.

Figure 2 shows a comparison of results from the centrifugal UF with data obtained during pressure-driven ultrafiltration through a $100 \mathrm{kDa}$ composite regenerated cellulose membrane (10). In this case, the data are plotted as a function of the average filtration velocity $(\bar{J})$, which was calculated as:

$$
\bar{J}=\frac{V}{A \bullet t} \quad[\text { Equation 1] }
$$

where $\mathrm{V}$ is the total collected filtrate volume during the centrifugation, $\mathrm{A}$ is the 
membrane area, and $t$ is the centrifugal time. The $y$-axis is the scaled filtrate concentration, equal to the plasmid concentration in the filtrate sample collected at the end of the centrifugation, divided by the arithmetic mean of the initial (feed) and final (retentate) plasmid concentrations in the solution above the membrane $(\bar{C})$. The results from the centrifugal and pressure-driven UF are in good qualitative agreement, with the data for the pressure-driven experiment shifted to slightly higher filtration velocities. In both cases, plasmid transmission was negligible below a critical filtration velocity between 40 and $60 \mu \mathrm{m} / \mathrm{s}$. Similar results were obtained with both smaller (3.0 and $5.9 \mathrm{~kb})$ and larger $(17$ $\mathrm{kb}$ ) plasmids, with the critical filtration velocity essentially independent of the plasmid size $(10,11)$.

Based on the results with the individual isoforms, a centrifugal UF experiment was performed to separate $450 \mu \mathrm{L}$ of a binary mixture containing $0.7 \mu \mathrm{g} / \mathrm{mL}$ of the supercoiled plasmid and $0.3 \mu \mathrm{g} / \mathrm{mL}$ of the linear isoform in $\mathrm{TE}$ buffer containing $10 \mathrm{mM} \mathrm{NaCl}$ (lane 2 in the agarose gel electrophoresis in Figure 3A). Data were obtained at 1350, 2350, and $2750 \mathrm{rpm}$, with the time adjusted to collect $160 \pm 10 \mu \mathrm{L}$ of filtrate. The supercoiled isoform migrates furthest through the gel, consistent with results from previous studies $(9,13)$. The faint band seen slightly above the linear isoform is likely due to the presence of a small quantity of a dimer of the supercoiled isoform or possibly trace amounts of the open-circular isoform initially present in the stock solution of the supercoiled plasmid. This species was fully retained by the membrane in all experiments.

The centrifugal UF at $1350 \mathrm{rpm}$ showed complete retention of both plasmid isoforms (Lane 4). The filtrate sample for the run at $2350 \mathrm{rpm}$ shows a significant amount of the linear isoform with only trace levels of the supercoiled isoform (Lane 6), consistent with the results shown previously in Figure 1. The use of a larger centrifugal speed $(2750 \mathrm{rpm})$ led to greater transmission of the linear isoform with a small but measureable amount of the supercoiled isoform collected in the filtrate (Lane 8). The bands corresponding to the supercoiled isoform in the final retentate samples (Lanes 3, 5, and 7) are significantly brighter than those in the feed (Lane 2), consistent with an increase in concentration of the highly retained supercoiled plasmid in these experiments.
Figure 3B shows corresponding results for centrifugal UF experiments with the same feed (Lanes 1 and 5) performed with higher degrees of filtration. Data were obtained at 2200 and $3000 \mathrm{rpm}$, providing $V=380$ and $360 \mu \mathrm{L}$ of filtrate (compared with the initial volume of 450 $\mu \mathrm{L})$; these experiments yielded average filtration velocity of $\bar{J}=37$ and $66 \mu \mathrm{m} / \mathrm{s}$, respectively. The filtrate samples (Lanes
4 and 7 ) show negligible amounts of the supercoiled isoform, consistent with operation near or below the critical average filtration velocity for transmission of this plasmid. The retentate samples (Lanes 3 and 6) show very bright bands for both isoforms due to the concentration of the retained plasmid.

The results in Figure 3B can be analyzed more quantitatively by integrating the

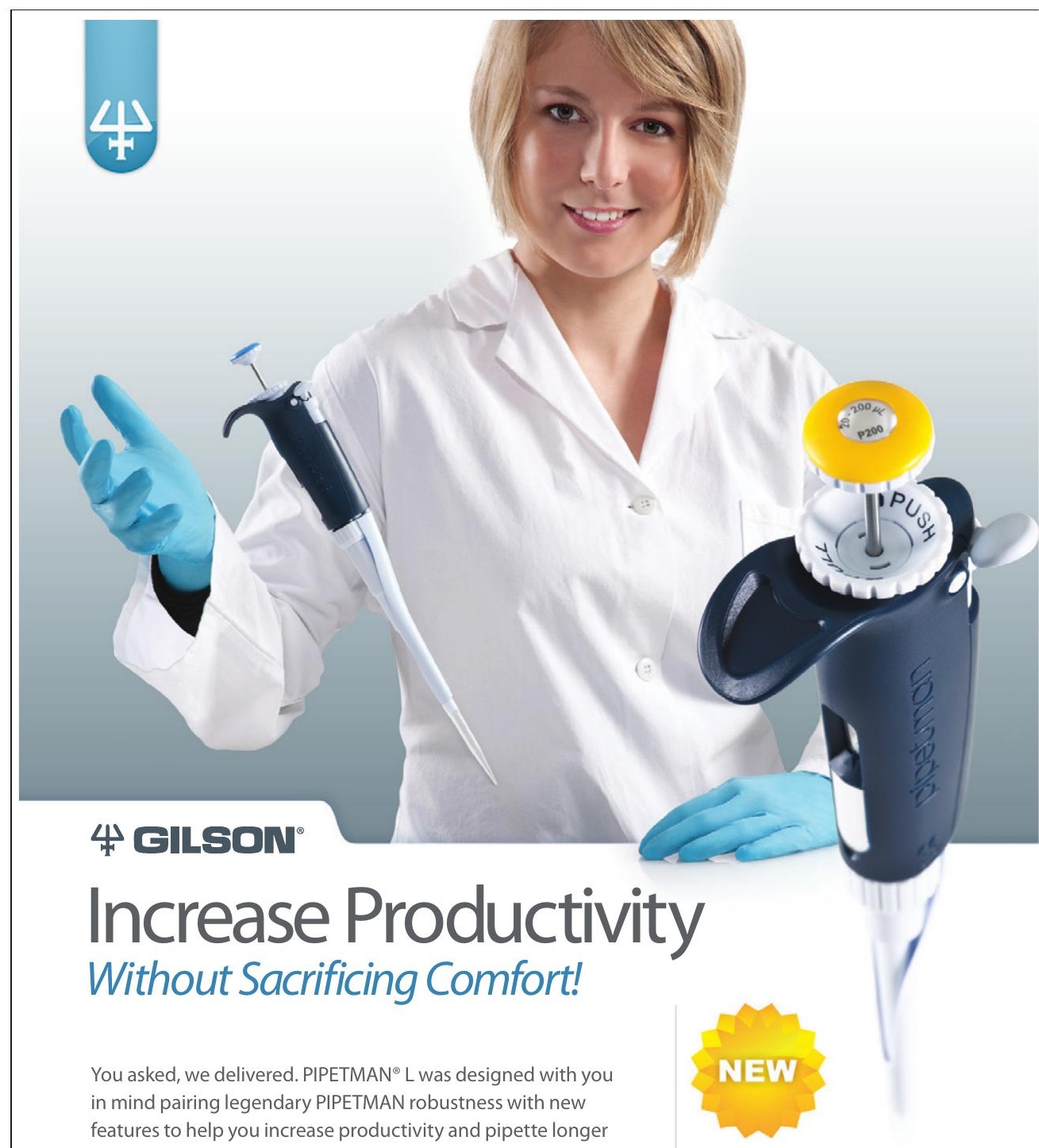

without sacrificing comfort.

凶 Lockable Volume Ensures No Accidental Volume Changes

$\otimes$ Lightweight Body for Comfortable Pipetting All Day

$\otimes$ Low Pipetting \& Tip Ejection Forces

\section{pipetman' $\mathrm{L}$}

www.PipetmanL.com 
A)

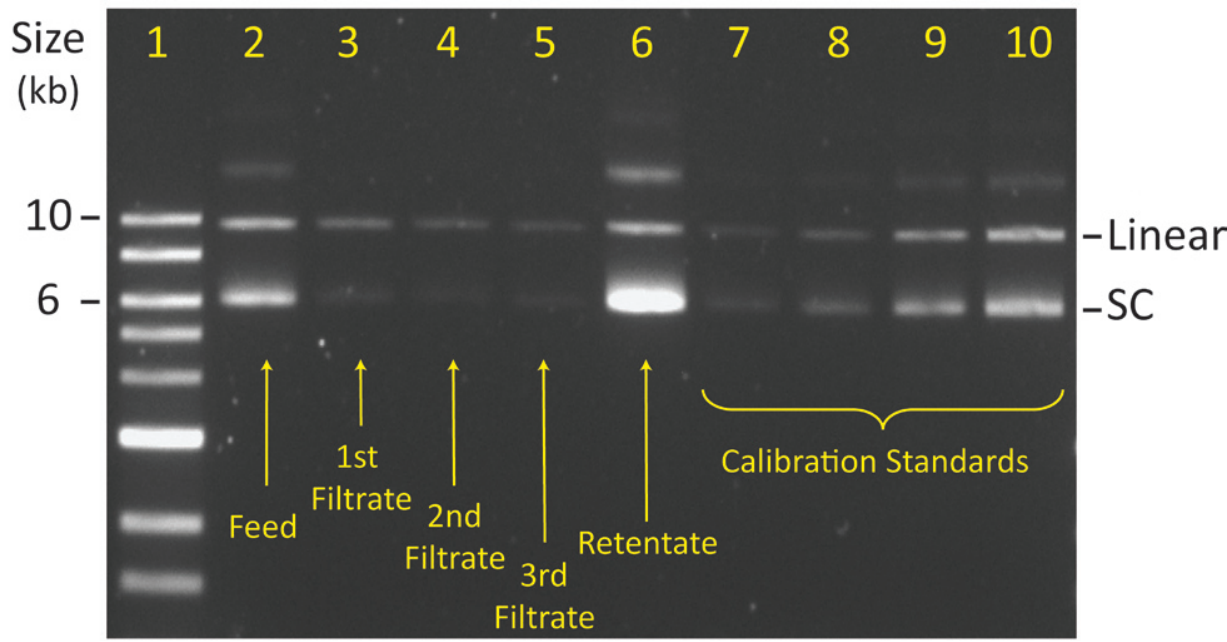

B )

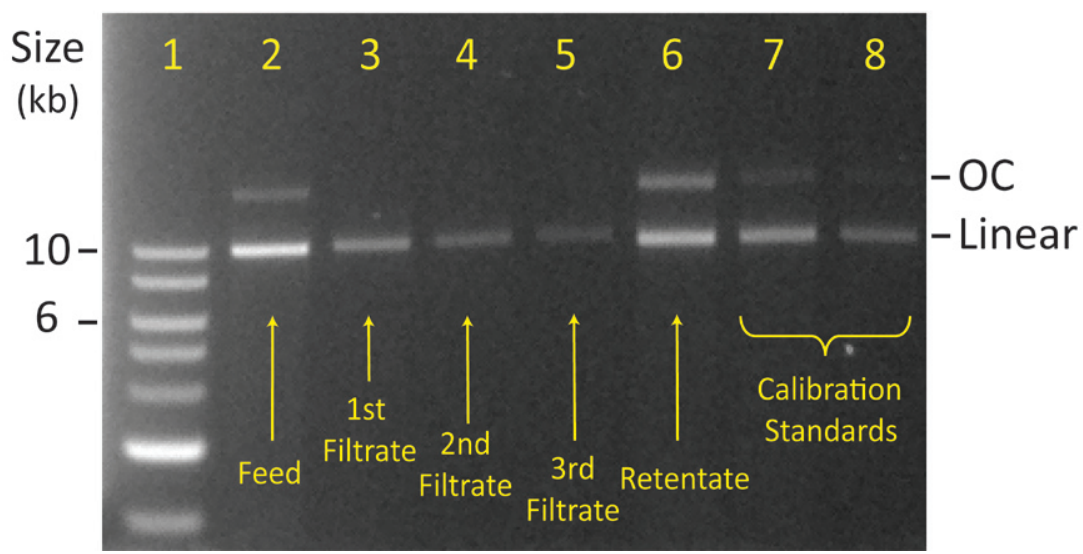

Figure 4. Separation of plasmid isoforms by diafiltration. Agarose gel electrophoregrams during three-step discontinuous diafiltrations of (A) mixture of linear and supercoiled (SC) isoforms and (B) mixture of linear and open-circular (OC) isoforms.

appropriate mass balances for an ultrafiltration process (14). The final expressions are conveniently expressed in terms of the observed sieving coefficient $\left(S_{o}\right)$, which is equal to the fractional transmission of the plasmid through the membrane. The plasmid concentrations in the final retentate $\left(C_{R}\right)$ and filtrate $\left(C_{f}\right)$ are given as (14):

$$
\begin{gathered}
\frac{C_{R}}{C_{o}}=\left(\frac{V_{o}}{V}\right)^{1-S_{o}} \quad \text { [Equation 2] } \\
\frac{C_{f}}{C_{o}}=\frac{1-\left(\frac{V}{V_{o}}\right)^{S_{o}}}{1-\left(\frac{V}{V_{o}}\right) \quad \text { [Equation 3] }}
\end{gathered}
$$

where $V / V$ is the concentration factor for the centrifugal UF. The predicted final retentate concentrations for the centrifugal UF experiments in Figure $3 \mathrm{~B}$ are $C_{R} / C_{0}=3.0$ and 4.6 using $S_{0}=$ 0.33 and $0.07^{\circ}$, respectively, for the linear and supercoiled isoforms at $3000 \mathrm{rpm}$. These values are consistent with the results in the AGE images based on the comparison of the intensity of the bands for the calibration standards (Lanes 9, 10, 11, and 12 in Figure $3 \mathrm{~A}$ and Lanes 8, 9, 10 , and 11 in Figure $3 B$ at concentrations of $1,0.5,0.2$, and $0.1 \mu \mathrm{g} / \mathrm{mL}$ ).

The purity of the retained (i.e., supercoiled) isoform can be increased by using a discontinuous diafiltration as described in the Methods. In this case, a threestep diafiltration was performed, with the feed volume reduced from $450 \mu \mathrm{L}$ to approximately $50 \mu \mathrm{L}$ during each UF step (followed by removal of the filtrate and re-dilution of the retentate to 450 $\mu \mathrm{L}$ using fresh TE buffer). Figure $4 \mathrm{~A}$ shows results for the separation of a mixture containing $0.3 \mu \mathrm{g} / \mathrm{mL}$ of the linear and $0.7 \mu \mathrm{g} / \mathrm{mL}$ of the supercoiled isoform (Lane 2) at $3000 \mathrm{rpm}$, providing an average filtration velocity of approximately $64 \pm 5 \mu \mathrm{m} / \mathrm{s}$. The filtrate solutions (Lanes 3, 4, and 5) are primarily the linear DNA isoform, with the concentration decreasing after each step in the discontinuous diafiltration as the linear DNA is removed from the centrifuge tube. The final retentate (Lane 6) has a bright band corresponding to the supercoiled DNA, with a relatively small amount of the remaining linear DNA, along with some DNA dimer, visible as distinct bands near the top of the gel.

The final concentrations of the linear and supercoiled DNA at the end of the discontinuous diafiltration can be calculated as (14):

$$
\frac{C_{R}}{C_{o}}=\left(\frac{V_{o}}{V}\right)^{1-n S_{o}}
$$

[Equation 4] 
where $\mathrm{n}$ is the number of steps in the discontinuous diafiltration $(\mathrm{n}=1$ corresponds to a simple ultrafiltration while $\mathrm{n}=2$ corresponds to a UF, dilution back to the initial volume, and then a second UF). If the observed sieving coefficients of the linear and supercoiled isoforms are assumed to be $S_{o}=0.33$ and 0.07 , respectively (consistent with the results in Figure 2), Equation 4 predicts that the final retentate would contain $3.6 \mathrm{\mu g} / \mathrm{mL}$ of the supercoiled plasmid and only 0.3 $\mu \mathrm{g} / \mathrm{mL}$ of the linear isoform (assuming a concentration factor of $V / V=8$ for each UF step), consistent with the images for the calibration standards. Further separation could be achieved using additional diafiltration steps. Model calculations indicate that the concentration of the linear isoform could be reduced by more than a 100 -fold using $\mathrm{n}=6$ diafiltration steps.

Corresponding results for the separation of a mixture of the open circular $(0.2 \mu \mathrm{g} /$ $\mathrm{mL}$ ) and linear $(0.8 \mu \mathrm{g} / \mathrm{mL})$ isoforms (Lane 2) are shown in Figure 4B. The discontinuous diafiltration was performed at $3300 \mathrm{rpm}$ yielding an average filtration velocity of $69 \pm 2 \mu \mathrm{m} / \mathrm{s}$. The filtrate samples for the 3 diafiltrations (Lanes 3, 4, and 5) contain only the linear isoform, leaving a final retentate solution (Lane 6) that is significantly more concentrated in the open circular isoform than the feed. Lanes 7 and 8 are calibration standards at total concentrations of 0.45 and $0.2 \mu \mathrm{g} / \mathrm{mL}$ ( $4: 1$ linear to open circular).

There are several distinct advantages to using centrifugal UF for plasmid separation. Centrifugal UF is fast, requiring only a few minutes compared with the several hours needed for agarose gel electrophoresis and density gradient centrifugation. Centrifugal UF requires no staining or visualization agents, eliminating the need to remove these dyes before subsequent processing steps. Centrifugal UF is readily performed using existing equipment and is easily incorporated into multi-step processes for DNA purification and handling. Scale-up can be achieved using larger volume centrifugal UF tubes; for example, Pall JumboSep tubes can handle up $60 \mathrm{~mL}$ of DNA solution. Note that separation of the supercoiled DNA from both the linear and open circular isoforms would require a multistep process: the linear DNA would first be removed using a discontinuous diafiltration at relatively low centrifugal speed, with the supercoiled plasmid then recovered in the filtrate using a second discontinuous diafiltration at higher centrifugal speed (Figure 1). The latter separation is more challenging because of the small difference in critical filtration velocity for the supercoiled and open circular isoforms and the presence of significant intermolecular interactions between these plasmids, particularly at higher concentrations. The model equations presented in the Supplementary materials provide a straightforward method for evaluating the appropriate centrifugal conditions.

\section{Acknowledgments}

This work was supported in part by grant CBET 0755816 from the National Science Foundation. The authors also acknowledge Dr. Jeff Chamberlain at the University of Washington for the $9.8 \mathrm{~kb}$ plasmid used in these studies, Dr. David Latulippe for helpful discussions, and Dr. Howard Salis for use of the centrifuge in his laboratory.

\section{Competing interests}

The authors declare no competing financial interests.

\section{References}

1. Blanco, S., C. Prat, M.A. Pallarés, L. Matas, and J. Domínguez. 2004. Centrifugal ultra-

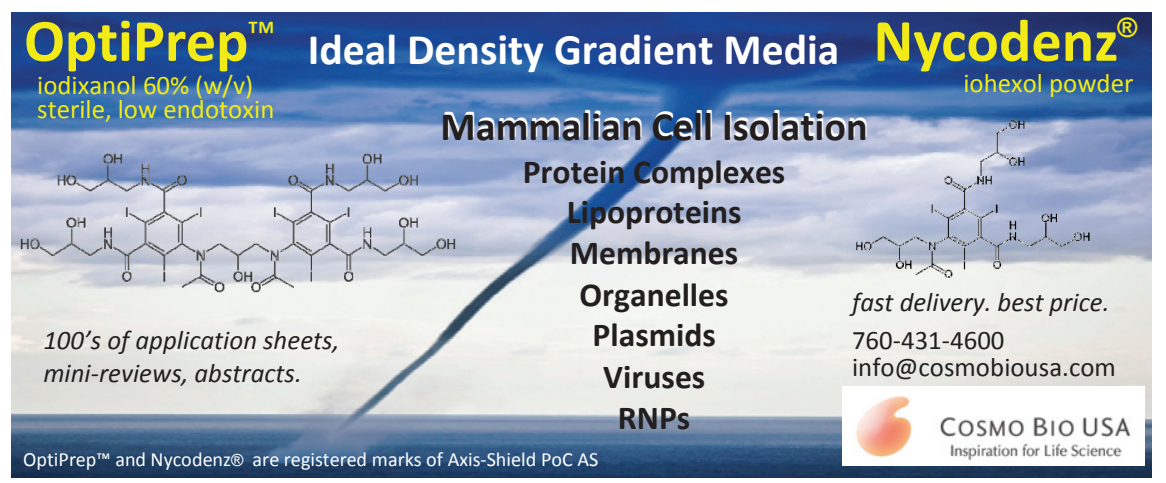

filtration method for rapid concentration of legionella pneumophila urinary antigen. J. Clin. Microbiol. 42:4410.

2. Liu, J., G.W. Lu, M. Sandoval, Y. Ciringh, G. Xue, D. Jaeger, K. Kompanik, J. Jiao, and K.M. Gelotte. 2009. Determination of benzalkonium chloride partition in micelle solutions using ultrafiltration method. AAPS PharmSciTech 10:1216-1223.

3. Greening, D.W. and R.J. Simpson. 2011. Low-molecular weight plasma proteome analysis using centrifugal ultrafiltration. Methods Mol. Biol. 728:109-124.

4. Lin, C.Y., C.H. Liu, H.C. Chang, and W.L. Tseng. 2008. Enrichment and separation of acidic and basic proteins using the centrifugal ultrafiltration followed by nanoparticlefilled capillary electrophoresis. Electrophoresis 29:3024-3031.

5. Sanchez, A.M., M. Carmona, M. Prodanov, and G.L.Alonso. 2008. Effect of centrifugal ultrafiltration on the composition of aqueous extracts of saffron spice (crocus sativus 1.). J. Agric. Food Chem. 56:7293-7301.

6. Krowczynska, A.M. and M.B. Henderson. 1992. Efficient purification of PCR products using ultrafiltration. Biotechniques 13:286289.

7. Schratter, P., A.M. Krowczynska, and J.T. Leonard. 1993. Ultrafiltration in molecular biology. Am. Biotechnol. Lab. 11:16.

8. McNevin, D., L. Wilson-Wilde, J. Robertson, J. Kyd, and C. Lennard. 2005. Short tandem repeat (STR) genotyping of keratinised hair part 2. an optimised genomic DNA extraction procedure reveals donor dependence of STR profiles. Forensic Sci. Int. 153:247-259.

9. Latulippe, D.R. and A.L. Zydney. 2010 Radius of gyration of plasmid DNA isoforms from static light scattering. Biotechnol. Bioeng. 107:134-142.

10. Latulippe, D.R., K. Ager, and A.L. Zydney. 2007. Flux-dependent transmission of supercoiled plasmid DNA through ultrafiltration membranes. J. Membr. Sci. 294:169-177.

11. Latulippe, D.R. and A.L. Zydney. 2009. Elongational flow model for transmission of supercoiled plasmid DNA during membrane ultrafiltration. J. Membr. Sci. 329:201-208.

12. Latulippe, D.R. and A.L. Zydney. 2011. Separation of plasmid DNA isoforms by highly converging flow through small membrane pores. J. Colloid Interface Sci. 357:548-553.

13. Moreau, N., X. Tabary, and F.L. Goffic. 1987. Purification and separation of various plasmid forms by exclusion chromatography. Anal. Biochem. 166:188-193.

14. Zeman, L.J. and A.L. Zydney. 1996. Microfiltration and ultrafiltration, principles and applications. Marcel Dekker, Inc., New York, NY.

Received 29 January 2012; accepted 13 June 2012.

Address correspondence to Andrew Zydney, Department of Chemical Engineering, Pennsylvania State University, University Park, PA 16802. Email: zydney@engr.psu.edu

To purchase reprints of this article, contact: biotechniques@fosterprinting.com 